\title{
Article \\ Adopting Feynman-Kac Formula in Stochastic Differential Equations with (Sub-)Fractional Brownian Motion
}

\author{
Bodo Herzog 1,2,3 (D)
}

Citation: Herzog, B. Adopting Feynman-Kac Formula in Stochastic Differential Equations with (Sub-)Fractional Brownian Motion. Mathematics 2022, 10, 340. https:// doi.org/10.3390/math10030340

Academic Editors: António M. Lopes, Alireza Alfi, Liping Chen and Sergio A. David

Received: 28 December 2021 Accepted: 21 January 2022

Published: 23 January 2022

Publisher's Note: MDPI stays neutral with regard to jurisdictional claims in published maps and institutional affiliations.

Copyright: (C) 2022 by the author. Licensee MDPI, Basel, Switzerland. This article is an open access article distributed under the terms and conditions of the Creative Commons Attribution (CC BY) license (https:// creativecommons.org/licenses/by/ $4.0 /)$.
1 Economics Department, ESB Business School, Reutlingen University, 72762 Reutlingen, Germany; Bodo.Herzog@Reutlingen-University.de

2 Reutlingen Research Institute (RRI), 72762 Reutlingen, Germany

3 Institute of Finance and Economics (IFE), Reutlingen University, 72762 Reutlingen, Germany

\begin{abstract}
The aim of this work is to establish and generalize a relationship between fractional partial differential equations (fPDEs) and stochastic differential equations (SDEs) to a wider class of stochastic processes, including fractional Brownian motions $\left\{B_{t}^{H}, t \geq 0\right\}$ and sub-fractional Brownian motions $\left\{\xi_{t}^{H}, t \geq 0\right\}$ with Hurst parameter $H \in\left(\frac{1}{2}, 1\right)$. We start by establishing the connection between a fPDE and SDE via the Feynman-Kac Theorem, which provides a stochastic representation of a general Cauchy problem. In hindsight, we extend this connection by assuming SDEs with fractionaland sub-fractional Brownian motions and prove the generalized Feynman-Kac formulas under a (sub-)fractional Brownian motion. An application of the theorem demonstrates, as a by-product, the solution of a fractional integral, which has relevance in probability theory.
\end{abstract}

Keywords: Cauchy problem; fractional-PDE; SDE; fractional Brownian motion; sub-fractional processes; Feynman-Kac formula; fractional calculus

\section{Introduction}

Consider the Cauchy problem [1] of the following parabolic partial differential equation (PDE) on $\mathbb{R}^{d}$

$$
\begin{aligned}
\frac{\partial}{\partial t} u(x, t) & =\kappa \frac{\partial^{2}}{\partial x^{2}} u(x, t)+\eta B^{H}(t), \quad t \geq 0, x \in \mathbb{R}^{d} \\
u(x, 0) & =u_{0}(x)
\end{aligned}
$$

where $u(x, t) \in C^{2,1}, u_{0}(x)$ is a bounded measurable function and $B^{H}(t)$ is a fractional Brownian motion (cf. Section 2). Without loss of generality, we assume that the parameter $\kappa$ is constant. This second-order PDE has a stochastic representation for $\eta=0$, according to the Feynman-Kac formula [2,3]. Indeed, we obtain

$$
u\left(x_{t}, t\right)=\mathbb{E}_{x, t}\left[u_{T}(x)\right]
$$

if $x_{t}$ satisfies Equation (3) and the function $\sigma\left(x_{t}, t\right)$ is sufficiently integrable

$$
d x_{t}=\mu\left(x_{t}, t\right) d t+\sigma\left(x_{t}, t\right) d B_{t}^{H},
$$

where $B_{t}^{H}$ is a Brownian motion (BM) if the Hurst parameter is of $H=\frac{1}{2}$ [4-6]. Additionally, the problem of (1) has an intimate relationship to the fractional partial differential equation (fPDE) [7]:

$$
\frac{\partial^{1 / 2}}{\partial t^{1 / 2}} u(x, t)=-\frac{\partial}{\partial x} u(x, t)
$$

Note that this equation contains a fractional derivative in general or a semi-derivative in respect of time in special [8-13]. 
There is a large amount of the literature devoted to each issue of the Cauchy problem $[6,14]$. This research closes a gap by considering the linking relationships of (sub-)fractional Brownian motions as well as fPDEs. The Feynman-Kac formula (2) provides a unique weak solution to Equation (1). Different versions of the Feynman-Kac formula have been discovered for a variety of problems $[15,16]$. Some generalizations of the Feynman-Kac formula are discovered by Querdiane and Silva [17] and Hu et al. [18,19]. A Feynman-Kac formula also exists for Lévy processes by Nualart and Schoutens [20].

Advancements in stochastic differential equations and fractional partial differential equations to analyse complex systems are related to our research [21-24]. Furthermore, recent developments in fractional calculus contributed to a better understanding and further studies of the relationships between fractional PDEs and stochastic calculus [25-31]. However, we are concerned about the linkage of the Cauchy problem and the representation by a fPDE, as well as the Feynman-Kac formula. For the Cauchy problem, we generalize the stochastic representation of Feynman-Kac by utilizing fractional Brownian motion (fBM) with Hurst parameter $H>1 / 2$.

In addition, the more recent literature looks at the idea of sub-fractional Brownian motion (sub-fBM). A sub-fBM is an intermediate between a Brownian motion and fractional Brownian motion. The existence and properties, such as long-range dependence, self-similarity and non-stationarity were introduced by Bojdecki et al. [32] and Tudor et al. $[33,34]$. Since the sub-fractional Brownian motion is not a martingale, methods of stochastic analysis are more sophisticated. However, several authors developed stochastic calculus and integration concepts for an fBM [25] and sub-fBM [35-37]. Recently, for a sub-fractional Brownian motion with Hurst parameters $H>\frac{1}{2}$, a maximal inequality was established according to the Burkholder-Davis-Gundy inequality for fractional Brownian motion [38]. It turns out that $\mathrm{fBM}$ and sub-fBM are adequate stochastic processes in scientific applications $[13,39]$.

In this paper, our purpose is to construct and prove a general link of the Cauchy problem with the Feynman-Kac equation via Itô's formula for $\mathrm{fBM}$ and sub-fBM. Consequently, this paper links the solution of $u(x, t)$ defined by Equation (1) with the stochastic Feynman-Kac representation to a fractional Brownian motion $\left\{B_{t}^{H}\right\}$ and sub-fBM $\left\{\xi_{t}^{H}\right\}$. We prove the result and show the properties of (sub-)fractional processes in stochastic analysis. Note that, throughout this paper, we frequently assume $\frac{1}{2}<H<1$.

The paper is organized as follows. Section 2 contains preliminaries on fractional calculus, particularly fractional Brownian motion. Thereafter, we examine sub-fractional stochastic processes and integration rules in Section 3. Here, we list the definitions and assumptions for the remainder of the article. In Section 4, we link the Cauchy problem to the Feynman-Kac formula with stochastic differential equations driven by fractional and sub-fractional Brownian motions. We state our theorems and prove our statements. In Section 5, we examine the Cauchy problem and the relationship to fractional partial differential equations (fPDE). Furthermore, we find a new fractional derivative and integral with relevance in probability theory. The conclusion is in Section 6.

\section{Preliminaries}

In the following section, we define preliminary concepts on fractional stochastic processes and fractional calculus.

\subsection{Fractional Calculus}

Since we deal with the Hurst parameter $H$, we need to know fractional calculus. Let $a, b \in \mathbb{R}, a<b$. Let $f \in L^{1}(a, b)$ and $\alpha>0$. The left- and right-sided fractional integral of $f$ of order $\alpha$ are defined for $x \in(a, b)$, respectively, as

$$
{ }_{a} D_{x}^{-\alpha} f(x)={ }_{a} I_{x}^{\alpha} f(x)=\frac{1}{\Gamma(\alpha)} \int_{a}^{x}(x-u)^{\alpha-1} f(u) d u \quad-\infty \leq a \leq x,
$$


and

$$
{ }_{x} D_{b}^{-\alpha} f(x)={ }_{x} I_{b}^{\alpha} f(x)=\frac{1}{\Gamma(\alpha)} \int_{x}^{b}(u-x)^{\alpha-1} f(u) d u \quad-\infty \leq x \leq b .
$$

This is the fractional integral of Riemann-Liouville type. Similarly, the fractional leftand right-sided derivative, for $f \in I_{a}^{\alpha}\left(L^{p}\right)$ and $0<\alpha<1$, are defined by

$$
{ }_{a} I_{x}^{-\alpha} f(x)={ }_{a} D_{x}^{\alpha} f(x)=\frac{1}{\Gamma(1-\alpha)}\left(\frac{d}{d x}\right) \int_{a}^{x}(x-u)^{-\alpha} f(u) d u
$$

and

$$
{ }_{x} I_{b}^{-\alpha} f(x)={ }_{x} D_{b}^{\alpha} f(x)=\frac{-1}{\Gamma(1-\alpha)}\left(\frac{d}{d x}\right) \int_{x}^{b}(u-x)^{-\alpha} f(u) d u,
$$

for all $x \in(a, b)$ and $I_{a}^{\alpha}\left(L^{p}\right)$ is the image of $L^{p}(a, b)$. It is easy to see that if $f \in I_{a}^{1}\left(L^{1}\right)$,

$$
{ }_{a} D_{x a}^{\alpha} D_{x}^{1-\alpha} f(x)=D f(x), \quad{ }_{b} D_{x b}^{\alpha} D_{x}^{1-\alpha} f(x)=D f(x) .
$$

Note $D^{\alpha} f(x)$ exists for all $f \in C^{\beta}([a, b])$ if $\alpha<\beta$.

\subsection{Fractional Stochastic Process}

Mandelbrot and van Ness defined a fractional Brownian Motion (fBM), $B_{t}^{H}$, as a Brownian motion, $B(t)$, together with a Hurst parameter (or Hurst index) $H \in(0,1)$ in 1968 [8]. The new feature of fBM's is that the increments are interdependent. The latter property is defined as self-similarity. A self-similar process has invariance with respect to changes in timescale (scaling-invariance). Almost all other stochastic processes, such as the standard Brownian Motion or Lévy processes, likely have independent increments. They create the famous class of Markov processes. Empirically, there is ubiquitous evidence in science that fractional stochastic processes, for instance, spectral densities with a sharp peak, are related to the phenomena of long-range interdependence over time. Indeed, the observable presence of interdependence in many real-world applications calls for fractional stochastic processes.

Definition 1. Let $H$ be $0<H<1$ and $B_{0}$ an arbitrary real number. We call $B^{H}(t, \omega)$ a fractional Brownian Motion ( $f B M)$ with Hurst parameter $H$ and starting value $B_{0}$ at time 0 , such as

(1) $B^{H}(0, \omega)=B_{0}$, and;

(2) $B^{H}(t, \omega)-B^{H}(0, \omega)=\frac{1}{\Gamma\left(H+\frac{1}{2}\right)}\left[\int_{-\infty}^{0}\left[(t-s)^{H-\frac{1}{2}}-(-s)^{H-\frac{1}{2}}\right] d B(s, \omega)+\int_{0}^{t}(t-s)^{H-\frac{1}{2}}\right.$

$d B(s, \omega)]$ [Wyle fractional integral];

(3) [Or equivalently by the Riemann-Liouville fractional integral: $B^{H}(t, \omega)-B^{H}(0, \omega)=$ $\left.\frac{1}{\Gamma\left(H+\frac{1}{2}\right)} \int_{0}^{t}(t-s)^{H-\frac{1}{2}} d B(s, \omega)\right]$.

We immediately obtain the corollary.

Corollary 1. For $H=\frac{1}{2}$ and $B_{0}=0$, we obtain a Brownian Motion $B(t, \omega)=B^{\frac{1}{2}}(t, \omega)$.

Proof. If $H=\frac{1}{2}$, we obtain $B^{\frac{1}{2}}(t, \omega)-B^{\frac{1}{2}}(0, \omega)=\frac{1}{\Gamma(1)} \int_{0}^{t} d B(s, \omega)=B(t, \omega)$.

For values of $H$, such as $0<H<\frac{1}{2}$ or $\frac{1}{2}<H<1$ the fBM $B^{H}(t, \omega)$ has different properties. If $0<H<\frac{1}{2}$, we say that it has the property of short memory. Indeed, Mandelbrot and van Ness [8] shows that this range is associated with negative correlation. If $\frac{1}{2}<H<1$, then the $\mathrm{fBM}$ has the property of long-memory or long-range dependence with time-persistence (Mandelbrot and van Ness [8]). Alternatively, we define a fractional Brownian motion by 
Definition 2. A fractional Brownian Motion ( $f B M)$ is a centered Gaussian process $B^{H}(t)$ for $t \geq 0$ with the covariance function

$$
R^{f B M}(t, s)=\mathbb{E}\left[B^{H}(t) B^{H}(s)\right]=\frac{1}{2}\left[|t|^{2 H}+|s|^{2 H}-|t-s|^{2 H}\right],
$$

where $H \in(0,1)$ denotes the Hurst parameter.

Remark 1. The covariance is trivially derived by starting with a standard Brownian motion and extending it with the Hurst index $H$, such as

$$
\begin{aligned}
\operatorname{Var}[B(t)-B(s)] & =\mathbb{E}\left[(B(t)-B(s))^{2}\right]=|t-s| \\
\Leftrightarrow \operatorname{Var}\left[B^{H}(t)-B^{H}(s)\right] & =\mathbb{E}\left[\left(B^{H}(t)-B^{H}(s)\right)^{2}\right]=|t-s|^{2 H},
\end{aligned}
$$

where, for $H=\frac{1}{2}$, we obtain the Brownian motion. The covariance is derived by the following steps

$$
\begin{aligned}
\operatorname{Cov}\left[B^{H}(t) B^{H}(s)\right] & =\mathbb{E}\left[\left(B^{H}(t)-\mathbb{E}\left[B^{H}(t)\right]\right)\left(B^{H}(s)-\mathbb{E}\left[B^{H}(s)\right]\right)\right]=\mathbb{E}\left[B^{H}(t) B^{H}(s)\right] \\
& =\frac{1}{2}\left[\mathbb{E}\left[B^{H}(t)^{2}\right]+\mathbb{E}\left[B^{H}(s)^{2}\right]-\mathbb{E}\left[\left(B^{H}(t)-B^{H}(s)\right)^{2}\right]\right] \\
& =\frac{1}{2}\left[|t|^{2 H}+|s|^{2 H}-|t-s|^{2 H}\right] .
\end{aligned}
$$

Corollary 2. The expectation of non-overlapping increments of an $f B M$ is $\mathbb{E}\left[B^{H}(t)-B^{H}(s)\right] \neq 0$ and the variance is of $\mathbb{E}\left[\left(B^{H}(t)-B^{H}(s)\right)^{2}\right]=|t-s|^{2 H}$ for all $t, s \in \mathbb{R}$

Proof. Let $t>s>0$. The first part is

$$
\begin{aligned}
\mathbb{E}\left[\left(B^{H}(t)-B^{H}(s)\right)\left(B^{H}(s)-B^{H}(0)\right)\right] & =\mathbb{E}\left[B^{H}(t) B^{H}(s)\right]-\mathbb{E}\left[B^{H}(t) B^{H}(0)\right]- \\
& -\mathbb{E}\left[\left(B^{H}(s)\right)^{2}\right]+\mathbb{E}\left[B^{H}(s) B^{H}(0)\right] \\
& =\frac{1}{2}\left[t^{2 H}+s^{2 H}-(t-s)^{2 H}\right]-s^{2 H} \\
& =\frac{1}{2}\left[t^{2 H}-s^{2 H}-(t-s)^{2 H}\right] \neq 0 .
\end{aligned}
$$

Thus, we can see that the expected increments are non-zero. Indeed, the increments are interdependent, contrary to Markov processes. The second part of the variance is

$$
\begin{aligned}
\mathbb{E}\left[\left(B^{H}(t)-B^{H}(s)\right)^{2}\right] & =\mathbb{E}\left[\left(B^{H}(t)-B^{H}(s)\right)\left(B^{H}(t)-B^{H}(s)\right)\right] \\
& =\mathbb{E}\left[\left(B^{H}(t)\right)^{2}\right]+\mathbb{E}\left[\left(B^{H}(s)\right)^{2}\right]-2 \mathbb{E}\left[B^{H}(t) B^{H}(s)\right] \\
& =t^{2 H}+s^{2 H}-2\left[\frac{1}{2}\left[|t|^{2 H}+|s|^{2 H}-|t-s|^{2 H}\right]\right] \\
& =|t-s|^{2 H} \quad \forall t, s \in \mathbb{R}
\end{aligned}
$$

Proposition 1. A fractional Brownian Motion ( $f B M)$ has the following properties:

(1) The fBM has stationary increments: $B_{t}^{H}-B_{s}^{H} \stackrel{\text { dis. }}{=} B_{u}^{H}-B_{s}^{H}$;

(2) The $f B M$ is H-self-similar, such as $B^{H}(a t)=a^{H} B^{H}(t)$;

(3) The fBM has dependence of increments for $H \neq \frac{1}{2}$. 
Proof. Part (1): For $t_{1}<t_{2}<t_{3}<t_{4}$, the equality of the covariance function implies that $Y:=B^{H}\left(t_{2}\right)-B^{H}\left(t_{1}\right)$ has the same distribution as $X:=B^{H}\left(t_{4}\right)-B^{H}\left(t_{3}\right)$. From above, we know

$$
\begin{aligned}
& \mathbb{E}\left[\left(B^{H}\left(t_{2}\right)-B^{H}\left(t_{1}\right)\right)^{2}\right]=\left(t_{2}-t_{1}\right)^{2 H}=(\Delta t)^{2 H} \\
& \mathbb{E}\left[\left(B^{H}\left(t_{4}\right)-B^{H}\left(t_{3}\right)\right)^{2}\right]=\left(t_{4}-t_{3}\right)^{2 H}=(\Delta t)^{2 H},
\end{aligned}
$$

where $t_{1}<t_{2}$ and $t_{3}<t_{4}$ with $\Delta t=t_{2}-t_{1}=t_{4}-t_{3}$. Hence, the incremental behavior at any point in the future is the same. Thus, we say that it has stationary increments.

Part (2): We show that $B^{H}(a t)=a^{H} B^{H}(t)$. We utilize the definition,

$$
\begin{aligned}
\mathbb{E}\left[\left(B^{H}(a t)\right)^{2}\right] & =\frac{1}{2}\left[(a t)^{2 H}+(a t)^{2 H}-(a t-a t)^{2 H}\right]=(a t)^{2 H}=a^{2 H} t^{2 H} \\
& =a^{2 H} \mathbb{E}\left[\left(B^{H}(t)\right)^{2}\right],
\end{aligned}
$$

hence, we obtain $\left(B^{H}(a t)\right)^{2}=a^{2 H}\left(B^{H}(t)\right)^{2}$ and this equal to $B^{H}(a t)=a^{H} B^{H}(t)$. The proof of part (3) is already in Corollary 2.

\subsection{Itô's Formula for Fractional Brownian Motion}

A fractional Brownian motion is continuous but almost certainly not differentiable [8]. Hence, it is inconvenient that an fBM does not have a derivative or integral. Furthermore, the $\mathrm{fBM}$ is neither a martingale nor a semi-martinagle. Therefore, Itô calculus is not applicable to fractional Brownian Motions if $H \neq \frac{1}{2}$.

However, stochastic calculus was developed with respect to fractional Brownian motion by [40] and the stochastic integral was introduced by [25]. The theory is a fractional extension of Itô-calculus, but limited to a Hurst index $H \in(1 / 2,1)$. If $H>1 / 2$ the fBM exhibits long-range dependence, which is a fundamental property in physics or finance.

By utilizing Wick calculus that has zero mean and explicit expressions for the second moment, we define the stochastic fractional integral, satisfying the property $\mathbb{E}\left[\int_{0}^{t} f(s) d B^{H}(s)\right]=0$.

Suppose a filtered probability space $\left(\Omega, \mathcal{F}, \mathbb{P}^{H}\right)$, where the probability measure depends on $H$. Note that $H$ is fixed by $H \in(1 / 2,1)$. Let us define a kernel function $\phi(s, t): \mathbb{R}_{+} \times \mathbb{R}_{+} \rightarrow \mathbb{R}_{+}$by

$$
\phi^{f B M}(s, t):=\phi(s, t)=H(2 H-1)|s-t|^{2 H-2} .
$$

Further, the functions $f$ and $g$ belong to the Hilbert space $L_{\phi}^{2}$ if

$$
|f|_{\phi}^{2}=\int_{0}^{\infty} \int_{0}^{\infty} f(s) f(t) \phi(s, t) d s d t<\infty,
$$

with the inner product defined by

$$
\langle f, g\rangle_{\phi}:=\mathbb{E}\left[\int_{0}^{\infty} f(s) d B^{H}(s) \int_{0}^{\infty} g(t) d B^{H}(t)\right]=\int_{0}^{\infty} \int_{0}^{\infty} f(s) g(t) \phi(s, t) d s d t
$$

This machinery leads to an analogue Itô formula for a fractional Brownian process. Already, Alòs et al. [41] proved this result under certain conditions for Itố's formula.

Theorem 1. (Alòs et al., 2001). Let $f$ be a function of class $C^{2}(\mathbb{R})$, satisfying the growth condition

$$
\max \left[|f(x)|,\left|f^{\prime}(x)\right|,\left|f^{\prime \prime}(x)\right|\right] \leq c e^{\left(\lambda|x|^{2}\right)},
$$

where $c$ and $\lambda$ are positive constants and $\lambda<\frac{1}{4} T^{-2 H}$. Suppose that $B^{H}=\left\{B_{t}^{H}, t \in[0, T]\right\}$ is a zero mean continuous Gaussian process whose covariance function $R^{f B M}(t, s)$ is of the form 
in Equation (8). Then, the process $F^{\prime}\left(B_{t}^{H}\right)$ belongs to a Hilbert space and, for each $t \in[0, T]$, the following Itô's formula holds:

$$
f\left(B_{T}^{H}\right)=f(0)+\int_{0}^{T} f^{\prime}\left(B_{s}^{H}\right) \delta B_{s}^{H}+\frac{1}{H} \int_{0}^{T} f^{\prime \prime}\left(B_{s}^{H}\right) s^{2 H-1} d s .
$$

However, we utilize a result by Duncan et al. [25], which is more convenient in our case. Here, is the Itô-Duncan theorem for a fractional Brownian motion:

Theorem 2. (Duncan et al., 2000, Thm 4.1, p. 596). If $f: \mathbb{R} \rightarrow \mathbb{R}$ is a twice continuously differentiable function with bounded derivatives to order two, i.e., $f \in C^{2}$, then

$$
f\left(B_{T}^{H}\right)-f\left(B_{0}^{H}\right)=\int_{0}^{T} f^{\prime}\left(B_{s}^{H}\right) d B_{s}^{H}+H \int_{0}^{T} s^{2 H-1} f^{\prime \prime}\left(B_{s}^{H}\right) d s \quad \text { a.s. }
$$

Remark 2. If $H=\frac{1}{2}$, we obtain, from Theorem 2, the usual Itô formula for a Brownian motion

$$
\begin{aligned}
f\left(B^{\frac{1}{2}}(T)\right)=f\left(B_{T}\right) & =\int_{0}^{T} f^{\prime}\left(B^{\frac{1}{2}}(s)\right) d B^{\frac{1}{2}}(s)+\frac{1}{2} \int_{0}^{T} s^{0} f^{\prime \prime}\left(B^{\frac{1}{2}}(s)\right) d s \\
& =\int_{0}^{T} f^{\prime}\left(B_{s}\right) d B_{s}+\frac{1}{2} \int_{0}^{T} f^{\prime \prime}\left(B_{s}\right) d s
\end{aligned}
$$

or in differential form

$$
d f\left(B_{T}\right)=f^{\prime}\left(B_{s}\right) d B_{s}+\frac{1}{2} f^{\prime \prime}\left(B_{s}\right) d s
$$

Similarly, for a function with two parameters $f\left(t, B_{t}^{H}\right)$, a generalized rule exists according to Duncan et al. [25].

Theorem 3. (Duncan et al., 2000, Thm 4.3, $p$. 596). Let $\eta_{t}=\int_{0}^{t} F_{u} d B_{u}^{H}$ for $t \in[0, T]$ and $\left(F_{u}, 0 \leq u \leq T\right)$ is a stochastic process in $\mathcal{L}(0, T)$. Let $f: \mathbb{R}_{+} \times \mathbb{R} \rightarrow \mathbb{R}$ be a function having the first continuous derivative in its first variable and the second continuous derivative in its second variable. Assume that these derivatives are bounded. Moreover, it is assumed that $\mathbb{E} \int_{0}^{T}\left|F_{s} D_{s}^{\phi} \eta_{s}\right| d s<\infty$ and $\left(f^{\prime}\left(s, \eta_{s}\right) F_{s}, s \in[0, T]\right)$ is in $\mathcal{L}(0, T)$. Then, for $0 \leq t \leq T$,

$$
\begin{aligned}
f\left(t, \eta_{t}\right)= & f(0,0)+\int_{0}^{t} \frac{\partial f\left(s, \eta_{s}\right)}{\partial s} d s+\int_{0}^{t} \frac{\partial f\left(s, \eta_{s}\right)}{\partial x} F_{s} d B_{s}^{H} \\
& +\int_{0}^{t} \frac{\partial^{2} f\left(s, \eta_{s}\right)}{\partial x^{2}} F_{s} D_{s}^{\phi} \eta_{s} d s \quad \text { a.s. }
\end{aligned}
$$

this is equal to

$$
d f\left(t, \eta_{t}\right)=\frac{\partial f\left(t, \eta_{t}\right)}{\partial t}+\frac{\partial f\left(t, \eta_{t}\right)}{\partial x} F_{t} d B_{t}^{H}+\frac{\partial^{2} f\left(t, \eta_{t}\right)}{\partial x^{2}} F_{t} D_{t}^{\phi} \eta_{t} d t,
$$

where $D_{s}^{\phi} \eta_{t}=\int_{0}^{t} D_{s}^{\phi} F_{u} d B_{u}^{H}+\int_{0}^{t} F_{u} \phi(s, u) d u$ a.s.

For the proof, we refer to Duncan et al. [25]. If $F(s)=a(s)$ is a deterministic function; then, the rule simplifies. Let $\eta_{t}=\int_{0}^{t} a_{u} d B_{u}^{H}$, where $a \in L_{\phi}^{2}$; then, we obtain

$$
\begin{aligned}
f\left(t, \eta_{t}\right)= & f(0,0)+\int_{0}^{t} \frac{\partial f\left(s, \eta_{s}\right)}{\partial s} d s+\int_{0}^{t} \frac{\partial f\left(s, \eta_{s}\right)}{\partial x} a(s) d B_{s}^{H} \\
& +\int_{0}^{t} \frac{\partial^{2} f\left(s, \eta_{s}\right)}{\partial x^{2}} \int_{0}^{s} \phi(s, v) a(v) d v d s \quad \text { a.s. }
\end{aligned}
$$

If $a_{s} \equiv 1$, then we obtain Itô's formula, such as in Theorem 2 and in Equation (13). 


\section{Sub-Fractional Stochastic Process}

A sub-fractional Brownian motion (sub-fBM) is an intermediate between a Brownian motion and fractional Brownian motion. It is a more general, self-similar Gaussian process or a generalization of a fBM. The sub-fBM has the property of H-self-similarity and longrange dependence, such as the fBM, yet it does not have stationary increments [32].

It is well-established that a stochastic process is uniquely determined by its covariance function $\operatorname{Cov}\left(\xi_{t}^{H}, \xi_{s}^{H}\right)$. Thus, we define:

Definition 3. A sub-fractional Brownian motion of Hurst parameter $H$ is a centered mean zero Gaussian process $\xi^{H}=\left\{\xi_{t}^{H}, t \geq 0\right\}$ with covariance function

$$
R^{s f B M}(t, s):=\mathbb{E}\left[\xi_{t}^{H} \xi_{s}^{H}\right]=s^{2 H}+t^{2 H}-\frac{1}{2}\left[(s+t)^{2 H}+|s-t|^{2 H}\right],
$$

where $\xi_{0}^{H}=0$ and $\mathbb{E}\left[\xi_{t}^{H}\right]=0$.

If $H=\frac{1}{2}$, it coincides with a Brownian motion on $\mathbb{R}_{+}$with covariance $\operatorname{Cov}\left(\xi_{t}^{H}, \xi_{s}^{H}\right)=$ $s \wedge t:=\min [s, t]$. The process $\xi_{t}^{H}$ has the following integral representation for $H>\frac{1}{2}$ (see [41]):

$$
\begin{aligned}
\xi_{t}^{H} & =\int_{0}^{t} K^{H}(t, s) d W_{s}, \\
K^{H}(t, s) & =c_{H}\left(H-\frac{1}{2}\right) s^{1 / 2-H} \int_{s}^{t}(u-s)^{H-3 / 2} u^{H-1 / 2} d u .
\end{aligned}
$$

Hence, the sub-fractional Brownian motion has a kernel of

$$
\phi^{s f B M}(s, t)=\frac{\partial^{2} \operatorname{Cov}\left(\xi_{t}^{H}, \xi_{s}^{H}\right)}{\partial s \partial t}=H(2 H-1)\left[|s-t|^{2 H-2}-(s+t)^{2 H-2}\right] .
$$

Note that the kernel has similarities to the fBM, as in Equation (9). Next, we discuss the main properties of a sub-fBM:

Lemma 1. Let $\xi_{t}^{H}$ be a sub-fBM for all $t$. It has the following properties:

(1) $\mathbb{E}\left[\left(\xi_{t}^{H}\right)^{2}\right]=\left(2-2^{2 H-1}\right) t^{2 H}$.

(2) $\mathbb{E}\left[\left(\xi_{t}^{H}-\xi_{s}^{H}\right)^{2}\right]=-2^{2 H-1}\left(t^{2 H}+s^{2 H}\right)+(t+s)^{2 H}+(t-s)^{2 H}$.

(3) If $H \neq \frac{1}{2}$, then $\xi_{t}^{H}-\xi_{s}^{H} \stackrel{\text { dis. }}{\neq} \xi_{u}^{H}-\xi_{s}^{H}$, i.e., the increments are non-stationary.

Proof. Part 1. Let $t=s$ in the covariance function $\operatorname{Cov}\left(\xi_{t}^{H}, \xi_{s}^{H}\right)$. We obtain $\operatorname{Cov}\left(\xi_{t}^{H}, \xi_{t}^{H}\right)=$ $\mathbb{E}\left[\xi_{t}^{2 H}\right]-\left(\mathbb{E}\left[\xi_{t}^{H}\right]\right)^{2}=\operatorname{Var}\left(\xi_{t}^{H}\right)$ and further we have $\operatorname{Var}\left(\xi_{t}^{H}\right)=\mathbb{E}\left[\left(\xi_{t}^{H}\right)^{2}\right]$ because $\xi_{t}^{H}$ is Gaussian with mean zero. Thus, using the covariance function in Definition 3, we obtain

$$
\mathbb{E}\left[\left(\xi_{t}^{H}\right)^{2}\right]=2 t^{2 H}-\frac{1}{2}(2 t)^{2 H}=2 t^{2 H}-\frac{1}{2}(2 t)^{2 H}=\left(2-2^{2 H-1}\right) t^{2 H} .
$$

Part 2. Given property 1 , one immediately obtains

$$
\begin{aligned}
\mathbb{E}\left[\left(\xi_{t}^{H}-\xi_{s}^{H}\right)^{2}\right] & =\left(2-2^{2 H-1}\right) t^{2 H}+\left(2-2^{2 H-1}\right) s^{2 H} \\
& =-2^{2 H-1}\left(t^{2 H}+s^{2 H}\right)+(t+s)^{2 H}+(t-s)^{2 H} .
\end{aligned}
$$

Part 3. Let $s=0$ and $t=h>0$, then $\mathbb{E}\left[\left(\xi_{h}^{H}-\xi_{0}^{H}\right)^{2}\right]=\mathbb{E}\left[\left(\xi_{h}^{H}\right)^{2}\right]=\left(2-2^{2 H-1}\right) h^{2 H}$ and we obtain 


$$
\begin{aligned}
\mathbb{E}\left[\left(\xi_{t+h}^{H}-\xi_{s+h}^{H}\right)^{2}\right] & =\mathbb{E}\left[\left(\xi_{2 h}^{H}-\xi_{h}^{H}\right)^{2}\right] \\
& =\mathbb{E}\left[\xi_{2 h}^{2 H}\right]-2 \mathbb{E}\left[\xi_{2 h}^{H}\right] \mathbb{E}\left[\xi_{h}^{H}\right]+\mathbb{E}\left[\xi_{h}^{2 H}\right] \\
& =\left(2-2^{2 H-1}\right)(2 h)^{2 H}+\left(2-2^{2 H-1}\right) h^{2 H}= \\
& =\left[2-2^{2 H-1}\right]\left(2^{2 H}+1\right) h^{2 H} .
\end{aligned}
$$

The difference in both increments is

$$
\Delta(H)=\left[2-2^{2 H-1}\right]-\left[2-2^{2 H-1}\right]\left(2^{2 H}+1\right)=-2^{2 H}\left[2-2^{2 H-1}\right],
$$

where $\Delta(H):=\mathbb{E}\left[\left(\xi_{h}^{H}\right)^{2}\right]-\mathbb{E}\left[\left(\xi_{t+h}^{H}-\xi_{s+h}^{H}\right)^{2}\right]$. For $\Delta(0)=-\frac{3}{2}$ and $\Delta\left(\frac{1}{2}\right)=-2$ and $\Delta(1)=0$. This implies that $\mathbb{E}\left[\left(\xi_{2 h}^{H}-\xi_{h}^{H}\right)^{2}\right]>\mathbb{E}\left[\left(\xi_{t}^{H}\right)^{2}\right]$ for all $H \in(0,1)$. Thus, the increments are non-stationary, such as $\xi_{t}^{H}-\xi_{s}^{H} \stackrel{\text { dis. }}{\neq} \xi_{u}^{H}-\xi_{s}^{H}$.

Finally, we prove two differences of fBM and sub-fBM.

Proposition 2. Let $B_{t}^{H}$ be a fractional Brownian motion and $\xi_{t}^{H}$ be a sub-fractional Brownian motion. For $H \in\left(\frac{1}{2}, 1\right)$ the following holds:

(1) $\mathbb{E}\left[\left(\xi_{t}^{H}\right)^{2}\right]<\mathbb{E}\left[\left(B_{t}^{H}\right)^{2}\right]$;

(2) $R_{\xi_{t}}(s, t) \leq R_{B_{t}^{H}}(s, t)$.

Proof. Part 1. For an $\mathrm{fBM}$, we have $\operatorname{Var}\left[B_{t}^{H}\right]=|t|^{2 H}$, and for the sub-fBM, we have $\operatorname{Var}\left[\xi_{t}^{H}\right]=\left(2-2^{2 H-1}\right)|t|^{2 H}$. Hence, we obtain $0<(2 H-1) \ln 2$ for $H>\frac{1}{2}$. For part 2, we show, under $s, t>0$, that

$$
\begin{aligned}
s^{2 H}+t^{2 H}-\frac{1}{2}\left[(s+t)^{2 H}+|t-s|^{2 H}\right] & \leq \frac{1}{2}\left[|t|^{2 H}+|s|^{2 H}-|t-s|^{2 H}\right] \\
s^{2 H}+t^{2 H} & \leq(s+t)^{2 H},
\end{aligned}
$$

where, only for $s=t=0$ or $s=0, t \neq 0$, we obtain equality.

\section{Itô's Formula for Sub-Fractional Brownian Motion}

For a Hurst parameter $H>\frac{1}{2}$, the stochastic integral of a sub-fBM $\int_{0}^{T} f(t) d \xi_{t}^{H}$ exists. The following theorem holds and is proven by [42]:

Theorem 4. Let $\xi_{t}^{H}$ be a sub-fBM defined in Definition 3 with $H>\frac{1}{2}$ and a function $f \in$ $L\left([0, T]^{2}, \phi^{s f B M} d \lambda_{2}\right)$, where $\lambda_{2}$ is a Lebesgue measure on $[0, T]^{2}$, where $\phi^{s f B M}(s, t)$ and $(s, t) \in$ $[0, T]^{2}$. Then, there exists a constant $C_{H}>0$ such that

$$
\mathbb{E}\left[\int_{0}^{T} f(t) d \xi_{t}^{H}\right]^{2} \leq C_{H}\|f\|_{L^{1 / H}\left([0, T], \lambda_{1}\right)}^{2} .
$$

According to Yan et al. ([36], Theorem 3.2 on p. 139) Itô's formula under a sub-fBM can be computed as follows:

Theorem 5. (Yan et al., 2011) Let $f \in C^{2}(\mathbb{R})$ and $H \in\left(\frac{1}{2}, 1\right)$. Then, we have

$$
f\left(\xi_{t}^{H}\right)=f(0)+\int_{0}^{T} f^{\prime}\left(\xi_{s}^{H}\right) d \xi_{s}^{H}+H\left(2-2^{2 H-1}\right) \int_{0}^{T} f^{\prime \prime}\left(\xi_{s}^{H}\right) s^{2 H-1} d s .
$$

Details of the proof are given in ([36], pp. 139-140). The authors even extend Itô's formula to $d$-dimensional sub-fBM and convex functions $f: \xi_{t}^{H} \rightarrow \mathbb{R}$. 


\section{Linking Cauchy via Feynman-Kac to SDEs with fBM and Sub-fBM}

Next, we derive the link between the Cauchy problem (1) and the stochastic representation according to Feynman-Kac by Equation (2). Consider a stochastic process $x_{s}$ on the time interval $[t, T]$ as the solution to the SDE in Equation (3). Next, use the Dynkin operator or Fokker-Planck operator $\mathcal{A}$ defined by

$$
\mathcal{A}=\mu(x, s) \frac{\partial}{\partial x}+\frac{1}{2} \sigma(x, s) \frac{\partial^{2}}{\partial x^{2}}
$$

We may write the Cauchy problem (1) as

$$
\begin{aligned}
\frac{\partial u(x, s)}{\partial s}+\mathcal{A} u(x, s) & =0, \\
u(x, T) & =u_{T}(x) .
\end{aligned}
$$

Cauchy Problem and Feynman-Kac

Applying Itô's lemma to $u(x, s)$. We obtain

$$
\int_{t}^{T} d u\left(x_{s}, s\right) d s=\int_{t}^{T}\left[\frac{\partial u\left(x_{s}, s\right)}{\partial s}+\mathcal{A} u\left(x_{s}, s\right)\right] d s+\int_{t}^{T} \sigma\left(x_{s}, s\right) \frac{\partial u\left(x_{s}, s\right)}{\partial x_{s}} d B_{s} .
$$

After integration, we obtain

$$
u\left(x_{T}, T\right)-u\left(x_{t}, t\right)=\int_{t}^{T}\left[\frac{\partial u\left(x_{s}, s\right)}{\partial s}+\mathcal{A} u\left(x_{s}, s\right)\right] d s+\int_{t}^{T} \sigma\left(x_{s}, s\right) \frac{\partial u\left(x_{s}, s\right)}{\partial x_{s}} d B_{s} .
$$

Since, by assumption $u(x, t)$ satisfies Equation (22), the time integral $d s$ in the last line of Equation (23) will vanish. Furthermore, if the process $\sigma\left(x_{s}, s\right) \frac{\partial u\left(x_{s}, s\right)}{\partial x_{s}}$ is sufficiently integrable, and after taking the expectation, the stochastic integral will vanish. Finally, considering the initial and boundary condition, such as $u(x, T)=u_{T}(x)$, we obtain the stochastic representation of the Cauchy problem (1) using the Feynman-Kac Formula (2) $[2,3]$ :

$$
u\left(x_{t}, t\right)=\mathbb{E}_{x, t}\left[u_{T}(x)\right] .
$$

Theorem 6. The stochastic representation of the Cauchy problem (1) under a generalized fractional Brownian Motion, $B_{t}^{H}$, with $H \in\left(\frac{1}{2}, 1\right)$, under the assumptions above, follows

$$
u\left(x_{t}, t\right)=\mathbb{E}_{x, t}\left[u_{T}(x)-\int_{t}^{T} \frac{\partial^{2} u\left(x_{t}, t\right)}{\partial x_{t}^{2}}\left[\int_{0}^{t} H f^{\prime \prime}\left(B_{v}^{H}\right) v^{2 H-1} d v\right] d s\right]
$$

and this simplifies under the conditions in Equation (14) to

$$
u\left(x_{t}, t\right)=\mathbb{E}_{x, t}\left[u_{T}(x)-\int_{t}^{T} \frac{\partial^{2} u\left(x_{t}, t\right)}{\partial x_{t}^{2}}\left[\int_{0}^{t} H(2 H-1)|t-v|^{2 H-2} a(v) d v\right] d s\right],
$$

if $x_{t} \in C^{2}$ and $\sigma\left(x_{t}, s\right)$ is independent of $x_{t}$. Note, for $H=\frac{1}{2}$, we obtain (2).

Proof. Consider $u\left(x_{t}, t\right)$ as solution of the Cauchy problem (1) under a generalized fractional Brownian Motion, $B_{t}^{H}$, with $H \in\left(\frac{1}{2}, 1\right)$. Applying Theorem 2 on $u(x, s)$, we obtain

$$
\begin{aligned}
\int_{t}^{T} d u\left(x_{s}, s\right) d s & =\int_{t}^{T}\left[\frac{\partial u\left(x_{s}, s\right)}{\partial s}+\mathcal{A} u\left(x_{s}, s\right)\right] d s+\int_{t}^{T} \sigma\left(x_{s}, s\right) \frac{\partial u\left(x_{s}, s\right)}{\partial x_{s}} d B_{s}+ \\
& +\int_{t}^{T} \frac{\partial^{2} f\left(x_{s}, s\right)}{\partial x_{s}^{2}}\left[\int_{0}^{t} H(2 H-1)|t-v|^{2 H-2} a(v) d v\right] d s
\end{aligned}
$$


After integration and under the assumption that $u(x, t)$ satisfies Equation (22). The time integrals will vanish. Given $x_{t} \in C^{2}$ and a deterministic $\sigma$, we obtain, after taking the expectation and the property that the stochastic integral vanishes, the stochastic representation as follows:

$$
u\left(x_{t}, t\right)=\mathbb{E}_{x, t}\left[u_{T}(x)-\int_{t}^{T} \frac{\partial^{2} u\left(x_{t}, t\right)}{\partial x_{t}^{2}}\left[\int_{0}^{t} H(2 H-1)|t-v|^{2 H-2} a(v) d v\right] d s\right] .
$$

If $H=\frac{1}{2}$, the stochastic representation simplifies to the well-known Feynman-Kac formula $u\left(x_{t}, t\right)=\mathbb{E}_{x, t}\left[u_{T}(x)\right]$.

Next, we state the Feynman-Kac formula for our Cauchy problem (1), given a subfractional Brownian motion.

Theorem 7. The stochastic representation of the Cauchy problem (1) under a sub-fractional Brownian Motion, $\xi_{t}^{H}$, with $H \in\left(\frac{1}{2}, 1\right)$ is

$$
u\left(x_{t}, t\right)=\mathbb{E}_{x, t}\left[u_{T}(x)-\int_{t}^{T} \frac{\partial^{2} u\left(x_{t}, t\right)}{\partial x_{t}^{2}}\left[\int_{0}^{t} H\left(2-2^{2 H-1}\right) f^{\prime \prime}\left(\xi_{v}^{H}\right) v^{2 H-1} d v\right] d s\right],
$$

if $x_{t} \in C^{2}$. Note, for $H=\frac{1}{2}$, we obtain the same as in Theorem 6 .

The proof follows an equal argument as above in the proof of Theorem 6.

\section{Cauchy Problem and Fractional-PDE}

Next, we demonstrate the direct linkage for the Cauchy-problem (1) to the fPDE in Equation (4). In step one, we compute the Laplace transform of the right-hand side of the heat equation:

$$
\begin{aligned}
\mathfrak{L}\left[u_{t}(x, t)\right] & =\mathfrak{L}\left[\frac{\partial u(x, t)}{\partial t}\right]=\int_{0}^{\infty} e^{-s t} \frac{\partial u(x, t)}{\partial t} d t \\
& =-u_{0}(x)+s \bar{u}(x, t) \\
& =s \bar{u}(x, t)
\end{aligned}
$$

where $\bar{u}(x, t):=\mathfrak{L}[u(x, t)]$. Thus, we obtain

$$
\begin{aligned}
\mathfrak{L}\left[\frac{\partial}{\partial x^{2}} u(x, t)\right] & =s \bar{u}(x, t) \\
\frac{\partial}{\partial x^{2}} \mathfrak{L}[u(x, t)] & =s \bar{u}(x, t) \\
\frac{\partial}{\partial x^{2}} \bar{u}(x, t) & =s \bar{u}(x, t) .
\end{aligned}
$$

This is a second-order ordinary differential equation in the $x$-variable. The solution is $\bar{u}(x, t)=c * e^{-\sqrt{s} x}$ for some constant $c$. Determining the constant by the second-derivative $\bar{u}_{x x}=c * s e^{-\sqrt{s} x}$ shows that $c=1$. In step two, we compute the first-derivative of the solution

$$
\begin{aligned}
\frac{\partial}{\partial x} \bar{u}(x, t) & =-\sqrt{s} e^{-\sqrt{s} x} \\
\frac{\partial}{\partial x} \bar{u}(x, t) & =-\sqrt{s} \bar{u}(x, t) .
\end{aligned}
$$


This is a first-order partial differential equation of the Laplace-transform $\bar{u}(x, t)$. Finally, compute the inverse Laplace transform and obtain the fPDE in Equation (4) by

$$
\frac{\partial}{\partial x} u(x, t)=-\frac{\partial^{\frac{1}{2}}}{\partial t^{\frac{1}{2}}} u(x, t) .
$$

Indeed, the inverse Laplace transform of the semi-derivative on the right-hand side is as follows:

$$
-\mathfrak{L}\left[\frac{\partial^{\frac{1}{2}}}{\partial t^{\frac{1}{2}}} u(x, t)\right]=u_{0}(x)-s^{\frac{1}{2}} \bar{u}(x, t)=-s^{\frac{1}{2}} \bar{u}(x, t)=-\sqrt{s} \bar{u}(x, t) .
$$

From the fractional representation of the Cauchy problem (1), we find the following fractional derivatives and integrals in relation to the normal distribution:

Proposition 3. Consider that the solution of the Cauchy problem (1) is of $u(x, t)=\frac{1}{\sqrt{2 \pi t}} e^{-\frac{x^{2}}{2 t}}$, which represents the normal probability density function $N^{\prime}(x)$ for a constant $t$. Thus, the solution of the fPDE (4) implies the following fractional derivative and integral:

(a) $\frac{\partial^{\frac{1}{2}}}{\partial t^{\frac{1}{2}}} u(x, t)=D_{t}^{\frac{1}{2}} u(x, t)=\frac{1}{\sqrt{2 \pi t}} \frac{x}{t} e^{-\frac{x^{2}}{2 t}}$.

(b) For $\alpha=\frac{1}{2}$, we find $I^{\alpha} u(x, t)=\frac{1}{\Gamma(\alpha)} \int_{-\infty}^{x}(x-t)^{\alpha-1} u(x, t) d t=N^{\prime}(x)$, where $N^{\prime}(x)$ is the density of the normal probability distribution in regard to $x$, or $N^{\prime}(x)=n(x)=\frac{1}{\sqrt{2 \pi t}} e^{-\frac{x^{2}}{2 t}}$.

Proof. Part (a): given $u(x, t)$, it follows from Equation (30) that the semi-derivative with respect to time $t$ is equal to $\frac{\partial}{\partial x} u(x, t)$. Computing the partial derivative of $u(x, t)$ with respect to $x$ is $u_{x}(x, t)=\frac{\partial u(x, t)}{\partial x}=\frac{1}{\sqrt{2 \pi t}} \frac{x}{t} e^{-\frac{x^{2}}{2 t}}$.

Part (b): In order to explicitly evaluate the fractional derivative, we utilize the linearity of both operators. Using operator calculus, we see that

$$
D_{t}^{\frac{1}{2}} u(x, t)=D_{t}^{1} D_{t}^{-\frac{1}{2}} u(x, t)=D_{t}^{1} I_{t}^{\frac{1}{2}} u(x, t) .
$$

Thus, the first-derivative of the semi-integral of $I_{t}^{\frac{1}{2}} u(x, t)$ with respect to $t$ must be equal to $u_{x}(x, t)$. Hence, the semi-integral

$$
I_{t}^{\frac{1}{2}} u(x, t)=\frac{1}{\Gamma\left(\frac{1}{2}\right)} \int_{-\infty}^{x}(x-t)^{\alpha-1} u(x, t) d t=N^{\prime}(x)=\frac{1}{\sqrt{2 \pi t}} e^{-\frac{x^{2}}{2 t}},
$$

consequently, the first-derivative of $N^{\prime}(x)$ is of $\frac{d N^{\prime}(x)}{d x}=N^{\prime \prime}(x)=\frac{1}{\sqrt{2 \pi t}} \frac{x}{t} e^{-\frac{x^{2}}{2 t}}$. The final term solves the fPDE in Equation (30). Thus, the fractional integral for $\alpha=\frac{1}{2}$ must be equal to the probability density function $N^{\prime}(x)$ in order to satisfy the fPDE in Equation (30).

\section{Conclusions}

This article studies the relationships of the Cauchy problem (1) and relates them to fractional partial-differential equations, as well as to the stochastic representations by the Feynman-Kac formula with a generalized fractional and sub-fractional Brownian motion with Hurst parameter $H>1 / 2$. In addition, we find fractional derivatives and integrals in relation to the Gaussian probability function by utilizing the novel insight into the linkage of the Cauchy problem and fPDE. This vantage point is of importance in probability theory, fractional calculus and stochastic theory. In future research, we intend to extend our theorems to Hurst parameters $H<1 / 2$ and the stochastic Cauchy problem under a sub-fBM. 
Funding: This research received funding from RRI-Reutlingen Research Institute.

Institutional Review Board Statement: Not applicable.

Informed Consent Statement: Not applicable.

Data Availability Statement: Not applicable.

Acknowledgments: The research of this paper was mainly finalized during my research semester 2021 at ESB Business School, Reutlingen University. I would like to express my thanks for the regular research semester and opportunity to advance scientific research for the good of the society in future.

Conflicts of Interest: The author declares no conflict of interest.

\section{References}

1. Kolodner, I. Free boundary problem for the heat equation with applications to problems of change of phase. Commun. Pure Appl. Math. 1956, 9, 1-31. [CrossRef]

2. Feynman, R.P. Space-time approach to nonrelativistic quantum mechansics. Rev. Mod. Phys. 1948, 20, 367-387. [CrossRef]

3. Kac, M. On distributions of certain Wiener functionals. Trans. Am. Math. Soc. 1949, 65, 1-13. [CrossRef]

4. Brown, R. A brief description of microscopical observations made in the months of June, July and August 1827, on the particles contained in the pollen of plants. Ann. Phys. 1828, 14, 294-313. [CrossRef]

5. Wiener, N. The average of an analytic functional and the Brownian movement. Proc. Natl. Acad. Sci. USA 1921, 7, $249-299$. [CrossRef]

6. Karatzas, I.; Shreve, S. Brownian Motion and Stochastic Calculus; Springer: Berlin/Heidelberg, Germany, 1991.

7. Babenko, Y. Teplomassoobmen. Metod Rascheta Teplovykh i Diffuzionnykh Potokov. (Heat and Mass Transfer: Calculating Heat and Diffusion Fluxes); Leningrad: Union City, NJ, USA, 1986.

8. Mandelbrot, B.; van Ness, J. Fractional Brownian Motions, Fractional Noises and Applications. SIAM Rev. 1968, 10, $422-437$. [CrossRef]

9. Oldham, K.; Spanier, J. The Fractional Calculus; Academic Press: New York, NY, USA, 1974.

10. Podlubny, I. Fractional Differential Equations; Elsevier: Amsterdam, The Netherlands, 1998; Volume 1.

11. Kilbas, A.; Srivastava, H.; Trujillo, J. Theory and Applications of Fractional Differential Equations; Elsevier: Amsterdam, The Netherlands, 2006; Volume 1.

12. Uchaikin, V. Fractional Derivatives for Physicists and Engineers; Springer: Berlin/Heidelberg, Germany, 2013.

13. Povstenko, Y. Fractional Thermoelasticity; Springer: Berlin/Heidelberg, Germany, 2015.

14. Walsh, J. An Introduction to Stochastic Partial Differential Equations; Springer: Berlin/Heidelberg, Germany, 1986; Volume XIV.

15. Ocone, D.; Pardoux, E. A stochastic Feynman-Kac formula for anticipating SPDEs, and application to nonlinear smoothing. Stoch. Rep. 1993, 45, 79-126. [CrossRef]

16. Mocioalca, O.; Viens, F. Skorohod integration and stochastic calculus beyond the fractional Brownian scale. J. Funct. Anal. 2005, 222, 385-434. [CrossRef]

17. Querdiane, H.; Silva, J.L. Generalized Feynman-Kac formula with stochastic potentials. Infin. Dimens. Anal. Quantum Probab. Relat. Top. 2002, 5, 243-255. [CrossRef]

18. Hu, Y.; Nualart, D.; Song, J. Feynman-Kac Formula for Heat Equation Driven by Fractional White Noise. Ann. Probab. 2011, 39, 291-326. [CrossRef]

19. Hu, Y.; Lei, F.; Nualart, D. Feynman-Kac Formula for Heat Equation Driven by Fractional White Noise with Hurst Parameter $H<1 / 2$. Ann. Probab. 2012, 40, 1041-1068.

20. Nualart, D.; Schoutens, W. Backward stochastic differential equations and Feynman-Kac formula for Lévy processes, with applications in finance. Bernoulli 2001, 7,761-776. [CrossRef]

21. Biagini, F.; Hu, Y.; Oksendal, B.; Zhang, T. Stochastic Calculus for Fractional Brownian Motion and Applications; Springer: Berlin/Heidelberg, Germany, 2008.

22. Embrechts, P. Selfsimilar Processes; Princeton University Press: Princeton, NJ, USA, 2002.

23. Nourdin, I. Selected Aspects of Fractional Brownian Motion; Springer: Berlin/Heidelberg, Germany, 2012.

24. Ruiz, W. Dynamical system method for investigating existence and dynamical property of solution of nonlinear time-fractional PDEs. Nonlinear Dyn. 2019, 99, 2421-2440.

25. Duncan, T.; Hu, Y.; Pasik-Duncan, B. Stochastic Calculus for Fractional Brownian Motion. SIAM J. Control. Optim. 2000, 38, 582-612. [CrossRef]

26. Marinov, T.M.; Ramirez, N.; Santamaria, F. Fractional Integration Toolbox. Fract. Calc. Appl. 2013, 16, 670-681. [CrossRef] [PubMed]

27. Fulinski, A. Fractional Brownian motions: Memory, diffusion velocity, and correlation functions. J. Phys. A Math. Theor. 2017, 50, 054002. [CrossRef]

28. Padhi, S.; Graef, J.; Pati, S. Multiple Positive Solutions for a boundary value problem with nonlinear nonlocal Riemann-Stieltjes Integral Boundary Conditions. Fract. Calc. Appl. Anal. 2018, 21, 716-745. [CrossRef] 
29. Kamran, J.W.; Jamal, A.; Li, X. Numerical Solution of Fractoinal-Order Fredholm Integrodifferentiantial Equation in the Sense of Atangana-Baleanu Derivative. Math. Probl. Eng. 2020, 2021, 6662803.

30. Guarigilia, E. Fractional calculus, zeta functions and Shannon entropy. Open Math. 2021, 19, 87-100. [CrossRef]

31. Sadhu, T.; Wiese, K. Functionals of fractional Brownian motion and three arcsine laws. arXiv 2021, arXiv:2103.09032.

32. Bojdecki, T.; Gorostiza, L.; Talarczyk, A. Sub-fractional Brownian motion and its relation to occuption times. Stat. Probab. Lett. 2004, 69, 405-419. [CrossRef]

33. Tudor, C. On the Wiener integral with respect to sub-fractional Brownian motion on an interval. J. Math. Anal. Appl. 2009, 351, 456-468. [CrossRef]

34. Tudor, C.; Zili, M. Covariance measure and stochastic heat equation with fractional noise. Fract. Calc. 2014, 17, 807-826. [CrossRef]

35. Shen, G.; Yan, L. The stochastic integral with respect to the sub-fractional Brownian motion with $H>\frac{1}{2}$. J. Math. Sci. 2010, 6, 219-239.

36. Yan, L.; Shen, G.; He, K. Itô's formula for a sub-fractional Brownian motion. Commun. Stoch. Anal. 2011, 5, 135-159. [CrossRef]

37. Liu, J.; Yan, L. Remarks on asymptotic behavior of weighted quadratic variation of subfractional Brownian motion. J. Korean Stat. Soc. 2012, 41, 177-187. [CrossRef]

38. Prakasa, R. On some maximal and integral inequailities for sub-fractional Brownian motion. Stoch. Anal. Appl. $2017,35,2017$.

39. Monin, A.; Yaglom, A. Statistical Fluid Mechansics: Mechanics of Turbulence; Dover Publication: Mineola, NY, USA, 2007; Volume II.

40. Decreusefond, L.; Üstünel, A. Stochastic analysis of the fractional Brownian motion. Potential Anal. 1998, 10, 177-214. [CrossRef]

41. Alòs, E.; Mazet, O.; Nualart, D. Stochastic Calculus with Respect to Gaussian processes. Ann. Probab. 2001, 29, 766-801. [CrossRef]

42. Mishura, Y.; Zili, M. Stochastic Analysis of Mixed Fractional Gaussian Processes; Mathematics and Statistics; Elsevier: Amsterdam, The Netherlands, 2018. 